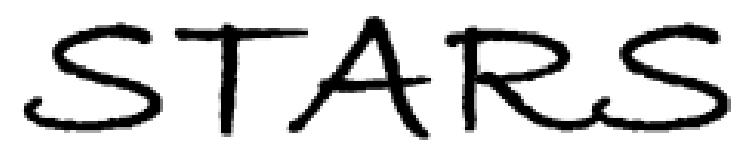

University of Central Florida

STARS

$1-1-2005$

\title{
Ultrashort, high-power pulse generation from a master oscillator power amplifier based on external cavity mode locking of a quantum-dot two-section diode laser
}

\author{
Myoung-Taek Choi \\ University of Central Florida \\ Wangkuen Lee \\ University of Central Florida \\ Ji-Myung Kim \\ University of Central Florida \\ Peter J. Delfyett \\ University of Central Florida
}

Find similar works at: https://stars.library.ucf.edu/facultybib2000

University of Central Florida Libraries http://library.ucf.edu

This Article is brought to you for free and open access by the Faculty Bibliography at STARS. It has been accepted for inclusion in Faculty Bibliography 2000s by an authorized administrator of STARS. For more information, please contactSTARS@ucf.edu.

\section{Recommended Citation}

Choi, Myoung-Taek; Lee, Wangkuen; Kim, Ji-Myung; and Delfyett, Peter J., "Ultrashort, high-power pulse generation from a master oscillator power amplifier based on external cavity mode locking of a quantumdot two-section diode laser" (2005). Faculty Bibliography 2000 s. 5067.

https://stars.library.ucf.edu/facultybib2000/5067

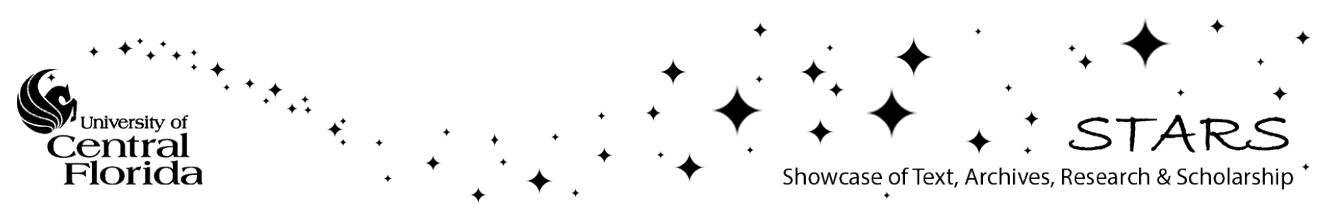




\title{
Ultrashort, high-power pulse generation from a master oscillator power amplifier based on external cavity mode locking of a quantum-dot two-section diode laser
}

\author{
Myoung-Taek Choi, ${ }^{a)}$ Wangkuen Lee, Ji-Myung Kim, and Peter J. Delfyett ${ }^{\text {b) }}$ \\ College of Optics and Photonics/CREOL \& FPCE, University of Central Florida, 4000 Central Florida \\ Boulevard, Orlando, Florida 32816
}

(Received 14 September 2005; accepted 24 October 2005; published online 22 November 2005)

\begin{abstract}
We investigate an external cavity curved two-section mode-locked diode laser system based on quantum-dot $(\mathrm{QD})$ gain media near $1.3 \mu \mathrm{m}$. Pulses are generated from the external laser cavity at a $5-\mathrm{GHz}$ repetition rate and amplified using a multilayer quantum-dot semiconductor optical amplifier. The pulses are compressed with a dual-grating dispersion compensator. The shortest, near transform-limited pulses are obtained when the mode-locked pulses are positive (up) chirped. The compressed pulses are $1.2 \mathrm{ps}$ in duration, with a pulse energy of $1.46 \mathrm{pJ}$, implying a peak power of 1.22 W. (c) 2005 American Institute of Physics. [DOI: 10.1063/1.2137309]
\end{abstract}

Monolithic mode-locked laser diodes (MMLDs) are devices in which entire optical cavity components, including gain and saturable absorber sections, are integrated together on a single chip. The MMLDs based on quantum-well (QW) and bulk structures have widely been studied ${ }^{1,2}$ due to their excellent performance in many application areas such as short pulse generation, high-bit rate optical telecommunication, microwave signal generation, ${ }^{3}$ and optical interconnects. 4

The MMLD based on quantum-dot (QD) gain media (QD-MMLD) is of interest owing to the improved performance expected, as compared to conventional QW/bulk devices. Intensive studies on QD-MMLD have been reported. $^{5-7}$ Their broad gain bandwidth and reduced linewidth enhancement factor make them excellent candidates as sources of ultrashort, ${ }^{8}$ low noise pulses. ${ }^{9}$ Because of its low internal absorption loss, the QD-MMLD becomes feasible at low repetition rates below $10 \mathrm{GHz}$, in contrast to conventional QW/bulk lasers. ${ }^{10}$ It should be noted that mode-locked operation of a QD monolithic device at a 5-GHz repetition rate has been demonstrated; ${ }^{9}$ however, lower repetition rates are difficult to achieve due to fabrication difficulty.

External cavity mode-locked semiconductor lasers (ECML) can be constructed out of a two-section device with a curved waveguide to reduce back reflection from a facet, and an external mirror. The ECML has many advantages over monolithic mode-locked lasers, such as repetition rate tunability and flexibility that allows the modification of the cavity design with other optical elements. The ECML also shows improvement in noise performance ${ }^{1}$ over MMLD because of the reduced length of the active waveguide for cavities of the same repetition rates. ${ }^{11}$

In this paper, we study an external cavity mode-locked QD two-section diode laser and the performance of external pulse amplification and compression. Ultrashort, high-power, and high-quality pulse trains are obtained after optical amplification with a multilayer high-gain quantum-dot semiconductor optical amplifier (QD-SOA) followed by a gratingbased dispersion compensator. The addition of the dispersion

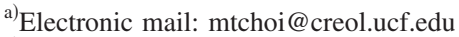

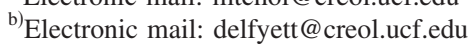

compensator provides access to both positive and negative dispersion, allowing the measurement of the sign and magnitude of pulse chirp.

The QD laser and amplifier epi structure was grown with molecular beam epitaxy (MBE) on an $n+$ GaAs substrate. The active region consists of 10 layers of self-assembled InAs/GaAs quantum dots, covered with $5 \mathrm{~nm} \mathrm{In}_{0.15} \mathrm{Ga}_{0.85} \mathrm{As}$. The two-section device used for this study consists of a gain section and a saturable absorber section. The gain section is curved and terminated at an angle of $7^{\circ}$ relative to the cleaved facet to minimize the back reflection from the facet. The residual reflectivity from the curved waveguide is estimated to be less than $10^{-5}$. $^{12}$ The device length is $3 \mathrm{~mm}$ with a 300- $\mu \mathrm{m}$-long saturable absorber (SA) section. A ridge waveguide of $5 \mu \mathrm{m}$ in width is formed by standard lithography and wet etching. The $15-\mu \mathrm{m}$-gap between two sections is chemically etched. The electrical isolation resistance between gain and absorber section was measured to be $6.4 \mathrm{k} \Omega$. After cleaving the device, it is mounted on a gold-coated copper stud with the $p$ side up, as cleaved.

To amplify the energy of pulses generated from ECML, a 3.2-mm-long semiconductor optical amplifier (SOA) was fabricated using the same wafer as the two-section device. The waveguide was angled by $7^{\circ}$, antireflection (AR) coated, and $p$ up mounted. Both the two-section device and SOA were kept at $20{ }^{\circ} \mathrm{C}$ using thermoelectric cooler control. The QD-SOA exhibited a thermal rollover over $300 \mathrm{~mA}$.

Using the curved two-section device and an output coupler of $70 \%$ reflectance, an external cavity was constructed. Figure 1 shows the schematic setup of the external laser cavity, the optical amplifier, and a grating compressor. The continuous-wave $(\mathrm{CW})$ threshold of the unbiased SA was $70 \mathrm{~mA}$. The light output versus forward bias current clearly shows a large hysterisis under reverse bias of the absorber. The pulses from the oscillator were amplified with the QDSOA. The maximum small signal gain and the output saturation power of the multilayer QD-SOA were measured to be $19 \mathrm{~dB}$ and $16 \mathrm{dBm}$, respectively, including coupling loss, which is comparable to the performance of conventional SOAs. The amplified pulses were diagnosed by an optical spectrum analyzer with $0.1 \mathrm{~nm}$ resolution, a $14-\mathrm{GHz}$ bandwidth photodetector followed by a microwave amplifier, a rf 


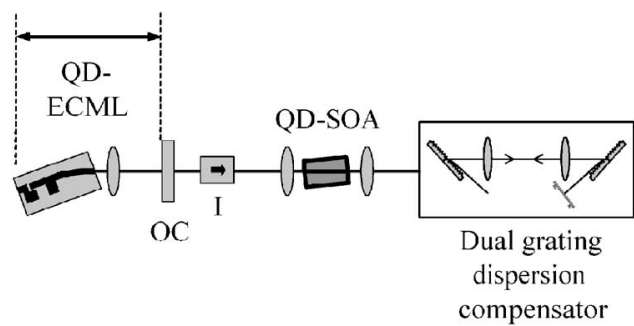

FIG. 1. Experimental setup. I: Isolator; OC: output coupler $(R=70 \%)$; QDECML: quantum-dot external cavity mode-locked laser; QD-SOA: quantum-dot semiconductor optical amplifier.

spectrum analyzer, a digital sampling scope, and a background-free autocorrelator.

Similar pulse widths and energy were obtained over a wide range of repetition rates, between 2.4 and $6 \mathrm{GHz}$. The fundamental cavity frequency is set to be $4.95 \mathrm{GHz}$ for further investigation. The average output power of $0.5-3 \mathrm{~mW}$ was obtained from the mode-locked laser cavity. Clean mode-locked pulses were generated without relaxation oscillation over a broad range of dc currents and reverse bias voltage. The full width at half maximum (FWHM) of the autocorrelation trace of the pulses generated from the oscillator varies from a few picoseconds to several tens of picoseconds depending on the bias condition. Figure 2 shows the pulse width change from 3.5 to $38 \mathrm{ps}$ as a function of reverse bias voltage at a dc current of $118 \mathrm{~mA}$. Near transform-limited pulses of 3.5 ps with $1.3 \mathrm{~nm}$ spectral width were obtained with a reverse bias voltage of $-8 \mathrm{~V}$. However, the pulse train quality degraded simultaneously, as manifested by the increased noise sidebands in the rf spectrum, and the pulse energy (average power) decreased significantly due to high reverse bias. In addition, multiple pulses were formed at this high reverse bias. Robust pulses with the lowest noise sidebands in the rf spectrum were obtained at a reverse bias of about $-5 \mathrm{~V}$ and a dc current of $118 \mathrm{~mA}$. Hybrid mode locking was performed by modulating the absorber section at the fundamental cavity frequency with a sinusoidal rf input power of $16-20 \mathrm{dBm}$. The pulse width was found to be similar in both hybrid and passive mode locking. The optical and rf spectra of the high quality passive mode-locked pulses are shown in Figs. 3(a) and 3(b).

A dual grating dispersion compensator was constructed to investigate the chirp of the mode-locked pulses. The internal telescope configuration was used to access both signs of chirp. The groove density of the grating was $1100 \mathrm{~g} / \mathrm{mm}$, and the insertion loss of the grating compensator after a double pass was $5.4 \mathrm{~dB}$. The mode-locked pulses with the autocorrelation signal width of 15 ps with $3 \mathrm{~dB}$ bandwidth of
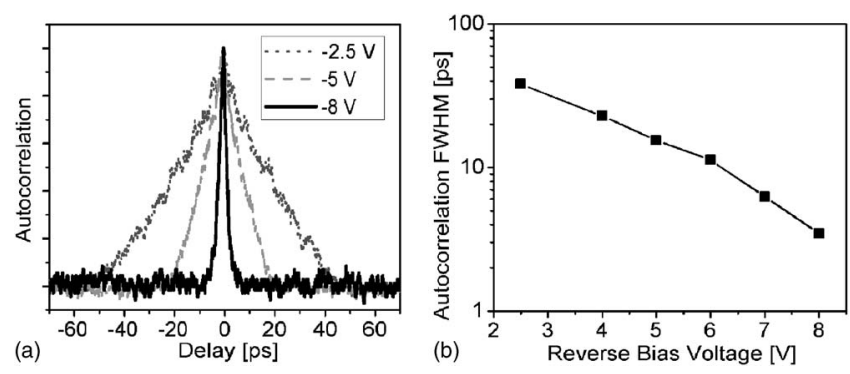

FIG. 2. (a) Normalized autocorrelation trace with different reverse bias voltage. (b) FWHM of autocorrelation signal vs reverse bias voltage on absorber section at $\mathrm{dc}=118 \mathrm{~mA}$.
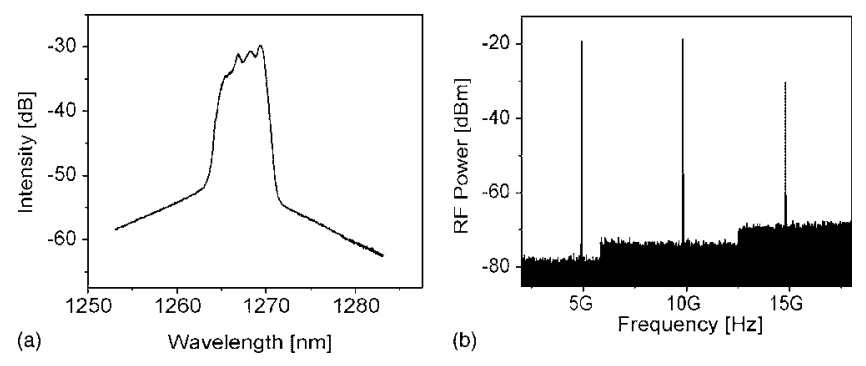

FIG. 3. Spectrum of passive mode-locked pulses. $\mathrm{dc}=118 \mathrm{~mA}$; reverse bias voltage $=-5 \mathrm{~V}$. (a) Optical spectrum, (b) rf spectrum.

the optical spectrum of $3.1 \mathrm{~nm}$ were sent to the grating compressor. The corresponding time bandwidth product (TBP) of $\sim 6$ implies that the mode-locked pulses generated from the ECML are highly chirped before compression. The linear component of the chirp is compensated by changing the position of the second grating. The optimum pulse compression was achieved in the negative GVD region of the compressor, where the position for the second grating is outside the focal length. Thus the output pulses of the ECML are positive or frequency up chirped. The up-chirped pulses are typically observed in electrically pumped passive mode-locked semiconductor lasers based on QW/bulk medium ${ }^{13}$ with only a few exceptions at very high repetition rate. ${ }^{14}$ As a result of the compression, 1.2-ps deconvolved pulse widths, assuming a $\operatorname{sech}^{2}$ pulse intensity profile are obtained. The TBP of 0.69 was achieved, which is about twice the Fourier-transform limit. Figure 4 shows the autocorrelation signal of the uncompressed and compressed pulses. The overlap between the experimental data and the fit shows the high quality of the compressed pulse shape without significant tails caused by nonlinear chirp. The average power after compression is $7.2 \mathrm{~mW}$, implying that the peak power and energy of the compressed pulses are $1.22 \mathrm{~W}$, and the $1.46 \mathrm{pJ}$, respectively.

To further investigate the chirp of the pulses generated from the laser cavity, we measured the magnitude of the chirp parameter as a function of driving currents and reverse bias voltages using a device with an absorber of $200 \mu \mathrm{m}$ long. The offset position of the second grating from zero dispersion providing the minimum pulse width is converted into the chirp parameter in units of $\mathrm{ps} / \mathrm{nm}$. Figure 5 shows that the magnitude of up chirp increases with injection

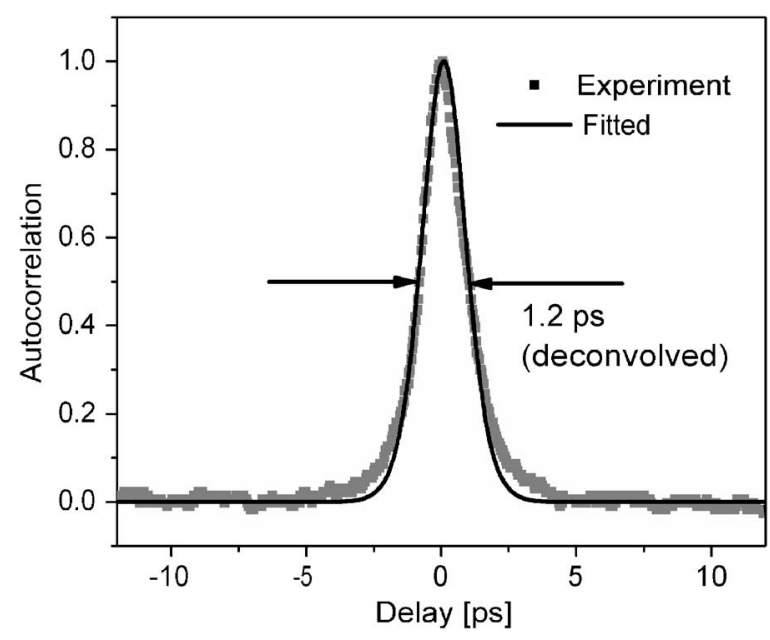

FIG. 4. Autocorrelation signal of the compressed pulses. 

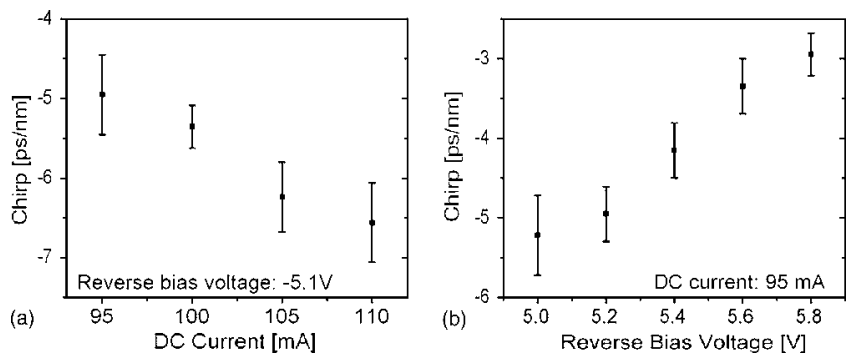

FIG. 5. The chirp of pulses as a function of (a) driving currents and (b) reverse bias voltages.

currents, and decreases with reverse bias voltages. The opposite trend is a consequence of the fact that the phase modulation of an absorbing media due to amplitude-phase coupling has the sign opposite to that of the gain medium. ${ }^{15}$ This measurement shows that the total chirp can be controlled by changing the relative strength of the absorber and gain sections. The two mechanisms may cancel each other under special conditions, leading to transform-limited pulses, ${ }^{5}$ which is most likely to occur at high reverse bias voltages. Another consequence of the large chirp measured in this experiment shows that the dynamic linewidth enhancement factor $(\alpha)$ of the QD device at high photon density is significantly large, in contrast to the small "material" $\alpha$ parameter below threshold, which is consistent with previous reports. ${ }^{16,17}$

In summary, the first external cavity mode-locked quantum-dot two-section laser is reported. It is found that the mode-locked pulses have strong linear chirp, enabling an order of magnitude compression ratio. Ultrashort pulses of $1.2 \mathrm{ps}$ width and a pulse energy of $1.46 \mathrm{pJ}$, implying a peak power of $1.22 \mathrm{~W}$, were obtained as a result of amplification with a multilayer QD-SOA and dispersion compensation. The measurement of chirp is reported for the first time in QD mode-locked lasers, highlighting the effect of the $\alpha$ parameter in saturable absorption and saturable gain.

${ }^{1}$ D. J. Derikson, R. J. Helkey, A. Mar, J. R. Karin, and J. G. Wasserbauer, IEEE J. Quantum Electron. 28, 2186 (1992).

${ }^{2}$ E. A. Avrutin, J. H. Marsh, and E. L. Portnoi, IEE Proc.: Optoelectron. 147, 251 (2000).

${ }^{3}$ T. Yilmaz, C. M. DePriest, P. J. Delfyett, Jr., T. Turpin, and J. H. Abeles, IEEE Photonics Technol. Lett. 14, 1608 (2002).

${ }^{4}$ G. A. Keeler, B. E. Nelson, D. Agarwal, C. Debaes, N. C. Helman, A. Bhatnagar, and D. A. B. Miller, IEEE J. Sel. Top. Quantum Electron. 9, 477 (2003).

${ }^{5}$ M. G. Thompson, K. T. Tan, C. Marinelli, K. A. Williams, R. V. Penty, I. H. White, M. Kuntz, D. Ouyang, D. Bimberg, V. M. Ustinov, A. E. Zhukov, A. R. Kovsh, N. N. Ledentsov, D. J. Kang, and M. G. Blamire, Electron. Lett. 40, 346 (2004).

${ }^{6}$ X. Huang, A. Stintz, L. Hua, L. F. Lester, I. Cheng, and K. J. Malloy, Appl. Phys. Lett. 78, 2825 (2001).

${ }^{7}$ M. Kuntz, G. Fiol, M. Lammlin, D. Bimberg, M. G. Thompson, K. T. Tan, C. Marinelli, R. V. Penty, I. H. White, V. M. Ustinov, A. E. Zhukov, Y. M. Shernyakov, and A. R. Kovsh, Appl. Phys. Lett. 85, 843 (2004).

${ }^{8}$ E. U. Rafailov, M. A. Cataluna, W. Sibbett, N. D. Il'inskaya, Yu. M. Zadiranov, A. E. Zhukov, V. M. Ustinov, D. A. Livshits, A. R. Kovsh, and N. N. Ledentsov, Appl. Phys. Lett. 87, 081107 (2005).

${ }^{9}$ L. Zhang, L. Cheng, A. L. Gray, S. Luong, J. Nagyvary, F. Nabulsi, L. Olona, K. Sun, T. Tumolillo, R. Wang, C. Wiggins, J. Zilko, Z. Zou, P. M. Varangis, H. Su, and L. F. Lester, Proceedings of Optical Fiber Communication, Anaheim, CA, March 6-11, 2005 (unpublished).

${ }^{10}$ F. Camacho, E. A. Avrutin, P. Cusumano, A. Saher Helmy, A. C. Bryce, and J. H. Marsh, IEEE Photonics Technol. Lett. 9, 1208 (1997).

${ }^{11}$ C. H. Henry, IEEE J. Quantum Electron. 18, 259 (1982).

${ }^{12}$ D. Marcuse, J. Lightwave Technol. 7, 336 (1989).

${ }^{13}$ M. Schell, J. Yu, M. Tsuchiya, and T. Kamiya, Appl. Phys. Lett. 67, 1797 (1995).

${ }^{14}$ R. A. Salvatore and A. Yariv, IEEE Photonics Technol. Lett. 7, 1151 (1995).

${ }^{15}$ G. P. Agrawal and N. A. Olsson, IEEE J. Quantum Electron. 25, 2297 (1989).

${ }^{16}$ A. Martinez, A. Lemaître, K. Merghem, L. Ferlazzo, C. Dupuis, A. Ramdane, J.-G. Provost, B. Dagens, O. Le Gouezigou, and O. Gauthier-Lafaye, Appl. Phys. Lett. 86, 211115 (2005).

${ }^{17}$ Hui Su and Luke F. Lester, J. Phys. D 38, 2112 (2005). 